\title{
SITUACION DE LA PISCICULTURA EN LA AMAZONIA PERUANA Y ESTRATEGIA PARA SU DESARROLLO
}

A lcántara Bocanegra Fernando ( $\left.{ }^{*}\right)$

\section{INTRODUCCION}

La piscicultura es una actividad productiva relativamente reciente en la amazonía peruana, con no más de tres décadas de desarrollo. Desde su inicio hasta hace unos cinco años se practicó en forma limitad a, principalmente a nivel familiar y durante los últimos años ha tenido un desarrollo acelerado, debido fundamentalmente a los avances obtenidos en el proceso de producción de semilla (alevinos) de las especie nativas: gamitana, Colossoma macropomum y paco Piractus brachypomus.

L os rendimientos que se están alcanzando en la actualidad son del orden de las 3.0 T.M./Ha/año, en la modalidad semi intensiva y de $7.0-9.0 \mathrm{~T} . \mathrm{M} . / \mathrm{Ha} / \mathrm{año}$, en la intensiva.

En este artículo se expone la situación actual de la piscicultura en la amazonía $y$, asimismo, se proponen estrategias para el desarrollo.

El autor expresa su agradecimiento al Blgo. Gilberto A scón Dionisio, investigador del IIAP en Tarapoto, por haber proporcionado información sobre infraestructura disponible para piscicultura en San M artín.

\section{ESTADO ACTUAL DE LA PISCICULTURA}

\subsection{Infraestructura disponible}

La capacidad instalada en toda la amazonía peruana se considera aún moderada; pero, a la vez, en franco proceso de crecimiento. El avance obtenido en la producción de semilla (alevinos), ha contribuido significativamente en su desarrollo y se prevee un crecimiento acelerado a medida que se obtengan mayores avances en la tecnología de cultivo y en la difusión regional de los alevinos. Por último, se observa que la inversión efectuada en este rubro es casi exclusiva del sector privado.

\subsubsection{Sector privado}

Según los registros de las Direcciones Regionales del $M$ inisterio de Pesquería en Iquitos, Pucallpa 'Tarapoto, se cuenta en la

\footnotetext{
Director General de Investigación en Hidrobiología (IIAP)
} 
actualidad con una superficie de 203.1 hectáreas de espejo de agua habilitado para el desarrollo de actividades de piscicultura en la amazonía peruana. De este total, el $56.5 \%$ está localizado en la R egión San Martín, el $26.4 \%$ en la Región U cayali y el $17,1 \%$ en la Región A mazonas (Cuadros N 2 1, 2, 3y 4).

\subsubsection{Sector estatal}

La infraestructura disponible a cargo del Estado en la amazonía peruana es del orden de 18.5 hectáreas (Cuadro № 5), equivalente al 9.1\% de la superficie disponible a nivel del sector privado.

\section{CUADRO №1}

\section{NUMERO DE ESTANQUES Y SUPERFICIE DISPONIBLE PARA PISCICULTURA EN LA REGION AMAZONAS Noviembre de 1990}

\begin{tabular}{|l|c|c|c|}
\hline \multicolumn{1}{|c|}{ PROVINCIAS } & No ESTANQUES & SUPERFICIE & $\%$ \\
\hline M aynas & 78 & 22.0 & 63.2 \\
A Ito A mazonas & 12 & 3.8 & 11.0 \\
L oreto & 22 & 7.0 & 20.1 \\
Requena & 2 & 0.5 & 1.4 \\
Ramón Castilla & 4 & 1.5 & 4.3 \\
\hline TOTAL & 118 & 34.8 & 100.0 \\
\hline
\end{tabular}

\section{CUADRO №2}

NUMERO DE ESTANQUES Y SUPERFICIE DISPONIBLE PARA PISCICULTURA EN LA REGION SAN MARTIN Noviembre de 1990

\begin{tabular}{|l|c|c|c|}
\hline \multicolumn{1}{|c|}{ PROVINCIAS } & No ESTANQUES & SUPERFICIE & $\%$ \\
\hline Rioja & 20 & 12.6 & 11.0 \\
M oyabamba & 51 & 19.4 & 16.9 \\
San M artin & 101 & 53.3 & 46.5 \\
Lamas & 18 & 3.1 & 2.7 \\
Bellavista & 12 & 2.7 & 2.3 \\
Huallaga & 7 & 5.0 & 4.3 \\
Mariscal Cáceres & 15 & 18.6 & 16.3 \\
\hline TOTAL & 224 & 114.7 & 100.0 \\
\hline
\end{tabular}

Fuente: Dirección Regional de Pesqueria (M oyabamba) 


\section{CUADRO №3}

NUMERO DE ESTANQUES Y SUPERFICIE DISPONIBLE PARA PISCICULTURA EN LA REGION UCAYALI

Noviembre de 1989

\begin{tabular}{|l|c|c|c|}
\hline \multicolumn{1}{|c|}{ PROVINCIAS } & No ESTANQUES & SUPERFICIE & $\%$ \\
\hline Carret. Federico Basadre & 57 & 38.6 & 72 \\
Carret. a Tournavista & 18 & 15.0 & 28 \\
\hline TOTAL & 75 & 53.6 & 100.0 \\
\hline
\end{tabular}

Reyes (1989)

\section{CUADRO №4}

NUMERO DE ESTANQUES Y SUPERFICIE DISPONIBLE PARA PISCICULTURA EN LA AMAZONIA PERUANA POR REGIONES

\begin{tabular}{|l|c|c|c|}
\hline \multicolumn{1}{|c|}{ REGION } & No ESTANQUES & SUPERFICIE. Ha. & $\%$ \\
\hline A mazonas & 118 & 34.8 & 17.1 \\
San M artin & 224 & 114.7 & 56.5 \\
U cayali & 75 & 53.6 & 26.4 \\
\hline TOTAL & 417 & 203.1 & 18.5 \\
\hline
\end{tabular}

\section{CUADRO №5}

NUMERO DE ESTANQUES Y SUPERFICIE DISPONIBLE PARA PISCICULTURA DE INSTITUCIONES ESTATALES EN LA AMAZONIA PERUANA

\begin{tabular}{|l|c|c|}
\hline \multicolumn{1}{|c|}{ INSTITUCION } & No ESTANQUES & SUPERFICIE. Ha. \\
\hline Instituto de Investigaciones de la A mazonía Peruana (IIAP)- & & \\
Iquitos (Investigación) & 20 & 2.0 \\
IIAP- Jenaro Herrera (Invest.) & 12 & 1.5 \\
IIAP- Pucallpa (Investigación) & 12 & 3.0 \\
Estación de Pesqueria A huashiyacu - Tarapoto (Fomento) & 239 & 8.0 \\
IVITA - Pucallpa (Investigación) & - & $4.0\left(^{*}\right)$ \\
\hline TOTAL & & \\
\hline
\end{tabular}

Cifra estimada

** Total sin considerar Pucallpa 


\section{$1.2 \quad$ Especies en cultivo}

\subsubsection{Especies nativas}

Las especies nativas de interés para el desarrollo de la piscicultura se vienen cultivando desde la década del sesenta. Los primeros trabajos se realizaron a cargo del Estado, en la Estación de Pesquería de Loreto. Estos primeros esfuerzos, sumados a los trabajos posteriores del Laboratorio de Iquitos del IMARPE y de la Estación de Pucallpa del Instituto Veterinario de Investigaciones Tropicales y de Altura (IVITA), permitieron seleccionar a las especies nativas de mayor potencialidad para la piscicultura, en la amazonia peruana, tales como: gamitana, Colossoma macropomun paco, Piaractus brachypomus boquichico Prochilodus nigricans sábalo cola roja, Brycon erythropterum, lisa, Schyzodon sp.

Es de esperarse que, a medida que avancen las investigaciones, se incremente el número de especies cultivadas; por el momento, se tienen observaciones preliminares sobre cultivo de otras especies, tales como: paiche A rapaima gigas arahuana Osteoglossum bicirrhossum.

Adicionalmente, se tienen aproximadamente cien especies de peces ornamentales que sustentan un activo comercio de exportación, reportándose la venta de un número aproximado a los 6’000,000 de ejemplares, por un valor de US \$700,000 para 1989 (Guerra y Col. 1990).

\subsubsection{Especies exóticas}

El interés por desarrollar la piscicultura en la amazonía peruana, así como el desconocimiento de la tecnología de cultivo de las especies nativas, determinó la necesidad de introducir especies de otros países, con tecnología probada. De esta manera se introdujeron varias especies de consumo; asimismo, se han introducido especies con otros fines, tanto para el control de los vectores de la malaria como para la ornamentación. A continuación se da una lista de las especies introducidas:

Especies de consumo: Tilapia, Sarotherodon hornorum; S. niloticus; $\underline{\text { S. aureum tilapia Tilapia rendalli }}$ carpa común, Cyprinus carpio carpa herbívora, Ctenopharingodon idella carpa plateada, Hypophtalmichtys molitrix. Camarón malasio, M acrobrachium rosenbergii. 
Especies para el control de los vectores de la malaria: gupis, lebistes recticulatus gambusia, Bambusia affinis. Especies ornamentales: beta, Betta splendens; gurami, Trichogaster trichopterus espada, Xiphophorus sp. y 'Gold Fish", Carassius sp.

Tanto las especies de consumo, como las especies para el control de los vectores de la malaria, fueron introducidas por entidades del Estado: M inisterio de Pesquería, M inisterio de Salud, IM ARPE y Universidades. Sin embargo, las ornamentales fueron introducidas por los exportadores de peces ornamentales de Iquitos, sin autorización y control del Estado. Este hecho, de por si grave, tiene una serie de implicancias ecológicas, tales como el riesgo de introducción de patógenos, potencialmente perjudiciales para las especies nativas, así como por el riesgo que significa el establecimiento de una especie exótica en el ecosistema amazónico.

Sobre el particular, se ha demostrado que las introducciones de especies exóticas conducen a los siguientes cuatro resultados posibles:

(1) "Extinción de homólogos ecológicos.

(2) Hibridación, con los efectos concomitantes en la genética de las poblaciones de peces originales.

(3) Fracaso de la introducción, originado en parte por la competencia con las especies establecidas.

(4) Coexistencia, lo que implica que la especie introducida ha encontrado un nicho vacante en la comunidad, con una segregación interactiva de nicho, como resultado obvio". (FAO/PNUMA, 1984).

En el caso de la amazonía peruana, se ha verificado el establecimiento de los gupis, L ebistes reticulatus la gambusia, Gambusia affinis y, recientemente, del gurami, Trichogaster trichopterus. Tanto los gupis, como la gambusia, se reproducen naturalmente en los canales de desague de la ciudad de Iquitos; en cambio, los guramis han alcanzado el río $\mathrm{N}$ anay, por lo menos en las zonas someras del río, próximas a Iquitos, su presencia es significativa frente a las especies nativas. Si bien no se ha llevado a cabo un estudio sistemático sobre esta última especie, se considera que por su sola presencia y reproducción natural en el $N$ anay, se amerita su evaluación.

El análisis de la información estadística del M inisterio de Pesquería - Dirección Regional de Iquitos, refleja que, durante el año 1979, se exportaron 19,800 ejemplares, por un valor declarado de US\$1,008 (Cuadro $\mathrm{N} 0$ 6), después de este año no se han registrado exportaciones, desconociéndose las causas. 


\section{CUADRO № 6}

EXPORTACION DE GURAMIS Trichogaster trichopterus

DESDE IQUITOS

\begin{tabular}{|l|c|c|}
\hline \multicolumn{1}{|c|}{ NOMBRE } & VOLUMEN & VALOR DECLA. \\
& & US\$ \\
\hline Blue gourami & 2,250 & 111.5 \\
Gold gourami & 12,000 & 309.0 \\
White gourami & 2,800 & 146.5 \\
Opalin gourami & 450 & 18.0 \\
Leeri Gourami & 2,300 & 123.0 \\
\hline Total & 19,800 & $1,008.0$ \\
\hline
\end{tabular}

FUENTE: MIPE (Iquitos)

\subsection{Disponibilidad de insumos para alimentación de peces.}

Entre los insumos disponibles a nivel regional se tienen una serie de productos, entre los que están los siguientes:

1.3.1 Harina de pescado. Es un producto altamente proteíco, que procede de la costa y es usado en alimentación de aves, porcina y vacuna. Principalmente se utiliza en la alimentación de aves y, en la última década, se empezó a utilizar también en la alimentación de peces, en forma restringida, en las instituciones del Estado que realizan investigación o fomento. Los inconvenientes del uso de este producto son su alto precio, así como su rápida al teración por la humedad.

No obstante lo anteriormente anotado, su disponibilidad es permanente a través de todo el año.

1.3.2 Torta de algodón. Es un producto con alto contenido de proteína y lípidos, procede de la costa o está disponible en Tarapoto. Al igual que el producto anterior, es utilizado en alimentación de aves, porcina y vacunos, principalmente; recientemente se usa en alimentación de peces. Su disponibilidad es permanente.

133 Torta de soya. Es un producto altamente proteíco que está disponible en Tarapoto, aunque su producción es escasa, por eso los criadores de aves lo importan de los Estados U nidos de N orte A mérica.

La disponibilidad del producto para alimentación animal, es escaso, en general; no obstante, la torta de soya ha sido utilizada en forma restringida, para la alimentación de peces. 
13.4 Harina de sangre. Es un producto con alto contenido de proteína que no está disponible comercialmente y que, por lo tanto, ha sido usado en forma restringida sólo en Iquitos, en alimentación de peces. Como insumo se ha utilizado la sangre de vacuno, colectada en el camal, la que actualmente se desperdicia.

13.5 Moyuelo de trigo. Es un subproducto energético que se obtiene en la producción de harina de trigo para pan; es utilizado en alimentación de aves, porcinos y vacunos, principalmente, y en forma restringida en la alimentación de peces, su disponibilidad es permanente a través de todo el año.

13.6 Maíz. Es un insumo energético de alta disponibilidad en la región y que es utilizado principalmente en alimentación de aves. Se ha utilizado también en alimentación de peces, pero en forma restringida. Se produce en toda la amazonía peruana.

1.3.7 Polvillo de arroz. Es un insumo energético que se obtiene en el pilado del arroz y es muy usado en alimentación de aves, porcinos y vacunos. Se utiliza también en alimentación de peces, pero en menor grado que en los anteriores. Ocasionalmente se presenta escasez del producto.

1.3.8 Otros productos. En adición a lo anteriormente anotado, en la alimentación de peces se utilizan productos agrícolas, como la yuca M anihot sculenta: industriales, corno los residuos de cervecería y, por último, productos del bosque, tales como: poma rosa, Psidium guajava mullaca, Physalia angulata: airambo, Phytolacarivinoides; cetico Cecropia sp.; pijuayo, Bactris gasipaes y míspero, A chras sapota.

Los productos del bosque citados se están utilizando en la alimentación de peces en la zona rural, en las inmediaciones de Iquitos, ya sea en forma exclusiva 0 combinados con polvillo de arroz.

\subsection{Produccción de alimento para peces}

La producción de alimento para peces con fines de cultivo itensivo es aún limitada y sólo se utiliza a nivel de las instituciones de investigación $y / 0$ fomento. Al nivel actual de desarrollo de la 
piscicultura, aún no se justifica la instalación de una planta de producción, ya que la mayoría de cultivos a nivel privado se realizan en forma extensiva (sin aporte de alimentos) o semi-intensiva (con aporte de abonos orgánicos e inorgánicos). Sin embargo, cabe señalar que durante los últimos años se viene observando un interés creciente por desarrollar el cultivo de peces a nivel privado, por lo que se prevée la necesidad de establecer plantas de producción de alimentos en Iquitos, Pucallpa y Tarapoto.

\subsection{Modalidades de cultivo y rendimientos}

Las modalidades predominantes de cultivo son la extensiva y la semi intensiva, en menor grado se desarrolla la modalidad intensiva; es decir, con aporte de alimento artificial.

A nivel de la amazonía peruana, se puede diferenciar dos modalidades predominantes, según localidades. En las zonas de I quitos y Pucallna, destacan los cultivos semi intensivos, con producciones del orden de 3.0 T.M./Ha/año, mientras que en la zona de Tarapoto predominan los cultivos intensivos con producciones de 7.0 - 9.0 T.M. $/ \mathrm{Ha} /$ año.

Lo anteriormente anotado se explica por la mayor disponibilidad de pescado procedente del medio natural, en Iquitos y Pucallpa y, consecuentemente, en la menor presión en la demanda para alimentación, en relación a la escasa y casi nula disponibilidad de pescado en la zona de Tarapoto y alta presión en la demanda. Adicionalmente, se tiene que en esta última zona existe mayor disponibilidad de insumos procedentes de actividades agrícolas e industriales para la alimentación de peces. Estas razones están favoreciendo un rápido desarrollo de la piscicultura, como una actividad productiva en San Martín, lo que se demuestra con la infraestructura habilitada en esta zona (Cuadro $\mathrm{N} \cong \mathrm{2}$ 2). L os rendimientos que se están alcanzando, así como avances en producción de alevinos en condiciones controladas en el IIAP, son los factores principales que están determinando un rápido desarrollo de la piscicultura en la amazonía del Perú, a esto se suma el esfuerzo constante desarrollado en las acciones de fomento por las Direcciones Regionales de San Martín, Pucallpa e Iquitos, del Ministerio de Pesquería.

De otro lado, se están desarrollando también cultivos asociados, tales como cerdos-peces y arroz-peces, pero en forma aún limitada y, en Tarapoto, se está cultivando el camarón gigante de agua 
dulce $M$ acrobrachium rosenbergii a nivel comercial. Al momento se desconocen los rendimientos que se están alcanzando. La situación antes descrita, está referida a los cultivos de especies de consumo; sin embargo, cabe señalar qu existe también el cultivo de paso', como se le llama al comercio de peces ornamentales, en el cual están operando, sólo en Iquitos, 25 empresas. Sobre el particular, cabe señalar que la exportación está basada exclusivamente en la extracción de las especies del medio natural y que la actividad se practica teniendo como común denominador los criterios tradicionales, más que los técnicos, por lo que los índices de mortalidad de las especies son elevados, habiéndose estimado niveles del orden del 60\% (Conroy, 1972). De otro lado, es también importante mencionar que, según las estadísticas de la Dirección Regional de Pesquería - Iquitos, durante los últimos tres años de la década se ha observado un incremento en el número de empresas dedicadas a este comercio en Iquitos y, contrariamente, se ha observado una disminución del número de ejemplares exportados, 10 que podría deberse a disminución en el control de la exportación 0 a disminución en la disponibilidad del recurso. (Guerra y Col., 1990).

\subsection{Personal involucrado en el desarrollo de la piscicultura}

\subsubsection{En cultivo de consumo}

A nivel del sector privado, se estima que, por lo menos, 4,000 a 5,000 personas están ligadas directamente a la piscicultura y, a nivel del sector estatal, se ha estimado que participan alrededor de 65 profesionales, entre personal de investigación, como de fomento, en toda la amazonía peruana. En relación a este

último punto, cabe señalar que se está observando escasez de personal profesional dedicado a la investigación en piscicultura, lo que se agrava por las restricciones legales vigentes para contratar personal y la escasa disponibilidad de recursos económicos destinados para la investigación. Si bien a la fecha se están realizando ya cultivos intensivos de las especies nativas, se considera que el estado de avance de esta tecnología requiere aún investigación para definir alternativas económicamente viables, que permitan alcanzar altas producciones en el menor tiempo posible.

1.6.2 En extracción y comercio de peces ornamentales

En la extracción y comercio de peces ornamentales se ha estimado que, a nivel privado, por lo menos diez mil personas participan directa e indirectamente, y que, a nivel estatal, participan 15 personas, entre profesionales y técnicos. 


\subsection{Instituciones involucradas en el desarrollo de la piscicultura en la amazonia peruana}

\subsubsection{Investigación}

En general, las investigaciones para el desarrollo de la piscicultura en la amazonia peruana están a cargo del Estado, a través del Instituto de investigaciones de la A mazonia Peruana (IIAP), o de las U niversidades, tales como la U niversidad $\mathrm{Nacional}$ de la A mazonia Peruana (UNAP), Universidad Nacional de Ucayali (UNU) Universidad Nacional Agraria de la Selva (UNAS), Universidad Nacional Mayor de San Marcos (UNMSM) y Universidad Nacional Agraria La Molina (UNA); estas dos ultimas de Lima. Dado el carácter autónomo de las instituciones de investigación que participan en este proceso, se observa con frecuencia descoordinaciones, dando como resultado la repetición de proyectos o experimentos, con el consiguiente desperdicio de infraestructura y recursos. En 1985, el IIAP propicié una reunión de coordinación interinstitucional, con la finalidad de ordenar el proceso de investigación en la amazonia, observándose, a la fecha, que se continúa repitiendo proyectos de investigación. Esta situación se agrava, aún más, por la carencia de planes nacionales y regionales de desarrollo de la actividad.

Como limitantes para el desarrollo de investigaciones en piscicultura se identifican, la escasez de personal profesional, así como la escasez de infraestructura disponible para esta tarea. Como se puede apreciar en el Cuadro №5, el mayor número de estanques para piscicultura del Estado, están destinados al fomento y en mucho menor grado a la investigación. En tal situación, desde 1984, el IIAP ha venido trabajando en convenio con el Ministerio de Pesquería Estación de Pesquería de Ahuashiyacu, para utilizar parte de la infraestructura de esa Estación en investigación. A la fecha continúa vigente el convenio, pero se están observando dificultades para su continuación $y$, de interrumpirse, se prevée un retroceso en la generación o adecuación de tecnología de cultivo de las especies y, por tanto, en el desarrollo de la actividad. De otro lado, cabe señalar que el estado de desarrolllo alcanzado en piscicultura, a nivel de la amazonía peruana, se debe fundamentalmente al esfuerzo nacional, ya que hasta ahora no se ha tenido apoyo directo ni de la cooperación técnica, ni financiera internacional. En todo caso, las entidades involucradas en este proceso llegan a Lima, la capital del país, posiblemente desconociendo que el Perú se encuentra en proceso de regionalización y que, en lo que se refiere a la región, es el Instituto de Investigaciones de la A mazonia Peruana (IIAP), con sede en Iquitos, el 
organismo responsable de la investigación de los recursos naturales, en general, y en particular de la piscicultura.

\subsubsection{Fomento}

El fomento de la acuicultura y más concretamente de la piscicultura en la amazonía del Perú, está a cargo de las Direcciones Regionales del Ministerio de Pesquería. Esta tarea, en general, es escasa y está enfocada mayormente a la etapa inicial del proceso; esto es, a la construcción de los estanques. El asesoramiento en cuanto a tecnología de cultivo de las especies es limitado, en parte por la escasez de personal técnico encargado de esta tarea, así como por la dispersión de las piscigranjas y los altos costos operativos de la extensión. En tal razón, se propone como alternativa para contrarrestar esta situación la realización de cursos de extensión, destinados a los piscicultores, previa capacitación y actualización de los extensionistas; en este sentido, debe propiciarse una coordinación estrecha entre las instituciones regionales de investigación y las de fomento, 0 en su defecto, encargar la tarea de extensión al personal dedicado a la investigación, ya sea en la etapa de divulgación de los avances 0 resultados alcanzados por la investigación, o en forma integral, como una tarea complementaria a la investigación.

En este punto, cabe señalar el rol trascendental que está cumtiliendo el Instituto de Investigaciones de la A mazonia Peruana (IIAP), en el desarrollo de la acuicultura en la región, ya que desde el año 1985 viene logrando la producción de crías de gamitana Colossoma macropomum y paco, Piaractus brachypomus en condiciones controladas para el fomento del cultivo de estas dos especies nativas. Si bien la tecnología de producción de semilla aún requiere investigación, el nivel de desarrollo alcanzado permite ya obtener rentabilidad en el proceso. Cabe señalar, asimismo, el rol importante que está desempeñando la Estación de Pesquería de A huashiyacu en San Martín, tanto por las acciones propias dcl asesoramiento técnico, como por la producción de alevinos de tilapia, especie introducida.

\subsubsection{Financiamiento}

Al estado actual de desarrollo de la piscicultura en la amazonía peruana, sólo el Banco Agrario participa como institución financiera. Sin embargo, cabe señalar que este apoyo es reciente y se otorga en forma limitada, previo estudio de factibilidad técnico-económica, preparado por los especialistas de las Direcciones Regionales de Pesquería. En la Región Ucayali, en adición a la participación del 
Banco Agrario, se tiene la contribución decidida del Gobierno Regional, que viene financiando conjuntamente con el IIAP, el Proyecto "Desarrollo de la piscicultura en Ucayali", con la finalidad de producir semilla (alevinos) de las especies nativas, tales como gamitana, Colossoma macropomun así como de la especie introducida tilapia, Q. niloticus. En la ejecución de este proyecto participan, además, el M inisterio de Pesquería, a través de su Dirección Regional de U cayali y el Instituto $V$ eterinario de Investigaciones Tropicales y de A Itura (IVITA). A dicionalmente, el Gobierno Regional de U cayali está apoyando la construcción de estanques en su ámbito, como una continuación de las acciones de la Ex-Corporación de Desarrollo (CORDEU). En el caso de lquitos el fomento realizado por la Dirección Regional de Pesquería, fue reforzado en la década del 80, inicialmente por la Ex-Corporación de Desarrollo de Loreto (CORDELOR) y posteriormente por el Gobierno Regional del Amazonas y el Gobierno Central, a través del Programa de Cooperación Popular. Finalmente, se está observando el interés creciente de los Consejos M unicipales Distritales y Provinciales, por desarrollar la piscicultura en su ámbito, como una actividad de interés social, para lo cual los propios alcaldes están participando directamente, así como en la asignación de fondos para adquisición de semilla.

\section{ESTRATEGIA PARA EL DESARROLLO DE LA PISCULTURA EN LA} AMAZONIA PERUANA

$2.1 \quad$ A mpliación de infraestructura

Para propiciar el crecimiento de la capacidad instalada para el desarrollo de la piscicultura en la A mazonia peruana, se debe procurar la integración de la actividad a las tareas agropecuarias, teniendo en cuenta que la instalación de la estanquería casi siempre se efectúa en los sectores cóncavos de los valles, dando como resultado un mejor uso de la tierra y, adicionalmente, el incremento en los ingresos que se obtienen de ella.

De otro lado, se deben establecer líneas de crédito específicas, con bajas tasas de interés, sobre todo para la línea de infraestructura y equipamiento.

\subsection{Especies y equipamiento}

Se debe impulsar el cultivo de las especies nativas, propiciando la profundización de las investigaciones para definir la tecnología del cultivo de las mismas. Al mismo 
tiempo, se debe procurar sustituir progresivamente el cultivo de las especies exóticas por las nativas, por los riesgos potenciales que representan para el ecosistema amazónico. De otro lado, se debe profundizar los estudios de biología básica de las especies hidrobiológicas nativas, para incorporar progresivamente otras especies a la piscicultura regional.

\subsection{Manejo de peces ornamentales}

Se considera necesario incorporar nuevas normas para la extracción, transporte y manejo de peces ornamentales en las instalaciones de exportación, las que deben estar a cargo de un profesional biólogo, a fin de introducir criterios técnicos en. El manejo de las especies y elevar los niveles de sobrevivencia en las diversas etapas del proceso. Adicionalmente, las Universidades de la Región deben reforzar la formación de profesionales biólogos con cursos de nutrición y patología de peces.

De otro lado, se deben otorgar incentivos para el establecimiento de laboratorios especializados en el diagnóstico de patógenos de peces ornamentales. De esta manera, se lograrían exportaciones de peces con certificación de libres de patógenos, hacia los mercados más exigentes del mundo que, por añadidura, pagan los mejores precios.

En relación a los peces ornamentales exóticos, se deben establecer prohibiciones terminantes para su introducción al país, salvo en casos plenamente justificados, en que previa opinión favorable de las instituciones de investigación del Sector Pesquero 0 del Instituto dc Investigaciones de la Amazonía Peruana (IIAP), se establecerían períodos de cuarentena para las especies exóticas por introducir, los que estarían a cargo de las instituciones antes mencionadas. Al mismo tiempo, se deben establecer responsabilidades para los organismos del Estado involucrados en la fiscalización del proceso de exportación.

\section{$2.4 \quad$ Investigación}

Se deben profundizar las investigaciones sobre cultivo y reproducción controlada de las especies nativas, tanto de consumo como ornamentales, priorizando los aspectos de nutrición, patología y manejo en condiciones controladas. 


\subsection{Alimento para peces}

Se deben realizar estudios de factibilidad técnicoeconómica para el establecimiento de plantas de producción de alimento para peces, en las localidades de Tarapoto, Iquitos y Pucallpa.

\section{$2.6 \quad$ Fomento}

En cuanto al fomento, se debe procurar que éste sea integral y que, además de orientarse a la construcción de estanques, se realice también la orientación precisa y oportuna en todo el proceso productivo. A fin de abaratar los costos de extensión, se deben organizar cursos periódicos sobre piscicultura.

\subsection{Cooperación técnica y financiera}

Se deben hacer esfuerzos para captar cooperación técnica y financiera internacional para las acciones de investigación, así como de fomento.

\section{BIBLIOGRAFIA}

CONROY, D.A. 1975. Una evaluación de la situación actual en el comercio mundial de peces ornamentales. Circular Pesquera de la FA O No. 335. Roma, Italia.

FA O/PNU M A 1984. Conservación de los recursos genéticos de los peces: problemas y recomendaciones. Informe de la consulta de expertos sobre los recursos genéticos de los peces. FAO Doc. Téc. Pesca, (217): 42p.

GUERRA, F.H.; F. ALCANTARA B.;. MACO G. y H. SANCHEZ R. 1990. La pesquería en el Amazonas Peruano, Interciencia. Vol. 15 No. 6 Caracas Venezuela.

REYES, A. W. 1989. Demanda de alevinos de gamitana (Colosomma macrotiunium) y boquichico (Prochilodus nigricans) para las Provincias de Coronel Portillo y Padre A bad, Región U cayali. Período 1990 - 1995. Informe Técnico No. 022-89PE/DIREPE VII/DAICT. Ministerio de Pesquería. Dirección Regional VII Pucallpa. 